\title{
GCU
}

Glasgow Caledonian

University

University for the Common Good

\section{Enhancing the error performance of optical SSK under correlated channel condition}

Popoola, Wasiu O.; Sinanovic, Sinan; Nistazakis, Hector E.

Published in:

2016 IEEE International Conference on Communications Workshops (ICC)

DOI:

10.1109/ICCW.2016.7503755

Publication date:

2016

Document Version

Author accepted manuscript

Link to publication in ResearchOnline

Citation for published version (Harvard):

Popoola, WO, Sinanovic, S \& Nistazakis, HE 2016, Enhancing the error performance of optical SSK under correlated channel condition. in 2016 IEEE International Conference on Communications Workshops (ICC). IEEE, pp. 7-11, 2016 IEEE International Conference on Communications Workshops (ICC), Kuala Lumpar, Malaysia, 23/05/16. https://doi.org/10.1109/ICCW.2016.7503755

\section{General rights}

Copyright and moral rights for the publications made accessible in the public portal are retained by the authors and/or other copyright owners and it is a condition of accessing publications that users recognise and abide by the legal requirements associated with these rights.

Take down policy

If you believe that this document breaches copyright please view our takedown policy at https://edshare.gcu.ac.uk/id/eprint/5179 for details of how to contact us. 


\title{
Enhancing the Error Performance of Optical SSK under Correlated Channel Condition
}

\author{
Wasiu O. Popoola, Sinan Sinanovic ${ }^{\dagger}$ and Hector E. Nistazakis $\ddagger$ \\ Institute for Digital Communications, School of Engineering, \\ University of Edinburgh, Edinburgh, EH9 3JL, UK. \\ $\dagger$ School of Engineering and Built Environment, \\ Glasgow Caledonian University, Glasgow, G4 0BA, UK. \\ $\ddagger$ Department of Electronics, Computers, Telecommunications and Control, Faculty of Physics, \\ National and Kapodistrian University of Athens, Athens, 15784, Greece \\ Email: \{w.popoola@ed.ac.uk, sinan.sinanovic@gcu.ac.uk and enistaz@phys.uoa.gr\}
}

\begin{abstract}
This paper presents ways of enhancing the performance of spatial shift keying (SSK) in optical wireless communication systems with correlated channel gains. One method involves varying the pulse width and the other involve varying the pulse amplitude to provide additional differentiation to the signal transmitted by each optical source. Closed form expressions are derived for the error performance and are validated with computer based simulations. When compared with the conventional SSK for the correlated channel gains under consideration, these enhancements methods could result in up to $20 \mathrm{~dB}$ reduction in signal-to-noise ratio at a symbol error ratio of $10^{-6}$ for a four-transmitter, one-receiver configuration.
\end{abstract}

\section{INTRODUCTION}

Various techniques exist in wireless communications to exploit the spatial position occupied by an antenna and/or receiver. Common ones include spatial diversity to provide resilience against debilitating channel conditions, spatial multiplexing and spatial modulation (SM) for increased throughput. These techniques have been extensively studied and reported in the listed literature and the references thereof [1]-[5].

When processing power is limited, the SM represents a low complexity multiple-transmitter approach to increase data rate [6], [7]. Using only the spatial position occupied by the transmitters to convey information is termed spatial shift keying (SSK) [8], [9] but adding another layer of signal constellation on to the SSK results in SM [10]. In the conventional SSK, only one transmitter is active during a given symbol duration. Also the number of transmitters in the conventional SSK is usually a power of two. Hence with a total of $N_{\mathrm{t}}$ transmitters, the SSK achieves $\log _{2} N_{\mathrm{t}}$ bits per symbol transmission from every transmitter.

Both SM and SSK rely primarily on the fact that the wireless communication channel gains vary with the relative spatial positions of the transmitters [5], [8], [11], [12]. In radio wireless communications, this is certainly the case in the presence of multiple waver scattering, reflection and node mobility. In optical wireless communications where there is no fast fading, the channel is rather static and the channel gains are more likely to be correlated than in radio wireless systems; especially when the transmitters are closely located. This motivates the work presented in this paper. Here, two approaches that can be implemented to enhance the performance of SSK in optical wireless communications systems with correlated channel gains are presented. The techniques involves adapting the properties of the pulse from each optical source to the channel conditions. In one of the enhancement approaches, the pulse width is varied while the amplitude is varied in the other to provide additional differentiation to the signal transmitted from each optical source. Although the emphasis in the work will be on SSK in indoor optical wireless communications, the methods discussed could also be applicable to SM.

The rest of the paper is organised as follows: the conventional SSK is briefly introduced in section II, the enhancement methods to make SSK adaptive are introduced in section III, results and discussions are presented in section IV while section $\mathrm{V}$ gives the concluding remarks.

\section{Optical SSK in CoRRelated Channel Conditions}

The optical SSK technique exploits the spatial dimension to increase spectral efficiency. It represents a low complexity multiple-input technique in which the spatial position of each optical source translates to a signal constellation point. The SSK with its $N_{\mathrm{t}}$ bits per symbols transmission compares with a pulse amplitude modulated (PAM) system with $N_{\mathrm{t}}$ distinct pulse amplitude levels. The difference here is that in the SSK transmitter, the transmitted pulses from all the LEDs have the same amplitude. Symbol differentiation and detection at the receiver is aided by the location dependent channel gains. Also, the SSK is simpler to implement compared to an identical PAM system [8]. With PAM, there is the risk of operating the LED beyond its dynamic range (into the nonlinear region) particularly as the number of levels increases. On the hand, SSK has only one signal level per LED, thus this risk can be easily eliminated with an increased number of LEDs. Also a single LED lamp often contain multiple light sources, this is what SSK exploits to increase data rate.

Consequently, the error performance of the SSK is strongly related to the difference between channel gains. For any given 
pair of channel gains $h_{i}$ and $h_{j}$, the error performance of an SSK system deteriorates considerably whenever these gains are correlated; that is $h_{i} \approx h_{j}$. This could be due to the close proximity of the optical sources. This is a possible scenario in visible light communications (VLC) when closely located multiple illumination LEDs are also used for wireless communication.

According to the work presented in [12], when there is strong correlation between any two given channel gains, it is almost impossible to establish a reliable optical wireless communication link based on optical SSK. This then led to the suggestions that SSK is best suited for a fixed configuration where the nodes are immobile and the channel gains are distinctive [12].

\section{ENCHANCED OPTICAL SSK IN CORRELATED CHANNEL}

To address the correlated channel problem, we will be considering the following two approaches to enhance the error performance of SSK in the this section.

\section{A. Optical SSK with Variable Pulse Width (eSSKv)}

An illustration of this scheme for the case of $N_{\mathrm{t}}=4$ is shown in Fig. 1. With this, 2 bits $\left(\log _{2} N_{\mathrm{t}}\right)$ can be transmitted during a symbol duration, same as the conventional SSK. In conventional SSK however, $\tau_{i}, i=1,2, \ldots N_{\mathrm{t}}$ is kept the same for all possible symbols. It is worthy of note that the variable width can be employed to convey additional bits (further increase the data rate) in an SM fashion. But here it used to provide resilience to channel correlation. By using the variable pulse width to provide an additional dimension for discerning the symbols, the error performance is thus improved while the data rate remains unchanged. Thus the duty cycle parameter $\tau_{i}$ becomes a design parameter that is assigned during the initial set-up stage, prior to the payload transmission. In some sense, this becomes a hybrid of SSK with pulse width modulation. In the event of clearly dissimilar channel gains, $\tau_{i}$ is chosen as the same for all $N_{\mathrm{t}}$. Otherwise, each transmitter has a different pulse duty cycle and the system resembles a pulse width modulated system. This offer an additional design flexible but at the cost of bandwidth expansion particularly for very small values of $\tau_{i}$.

For an $N_{\mathrm{t}}$-transmitter and $N_{\mathrm{r}}$-receiver OWC configuration, the received signal can be expressed as:

$$
\mathbf{r}(\mathbf{t})=R \mathbf{H}(\mathbf{t}) \otimes \mathbf{x}(\mathbf{t})+\mathbf{n}(\mathbf{t})
$$

where $\otimes$ denotes convolution operation and $\mathbf{n}(\mathbf{t})$ is the $N_{\mathrm{r}^{-}}$ dimensional noise vector. The noise is the sum of the receiver thermal noise and ambient light; it is modelled as independent and identically distributed additive white Gaussian noise (AWGN) while $\mathbf{x}(\mathbf{t})$ is the $N_{\mathrm{t}}$-dimensional data signal. The

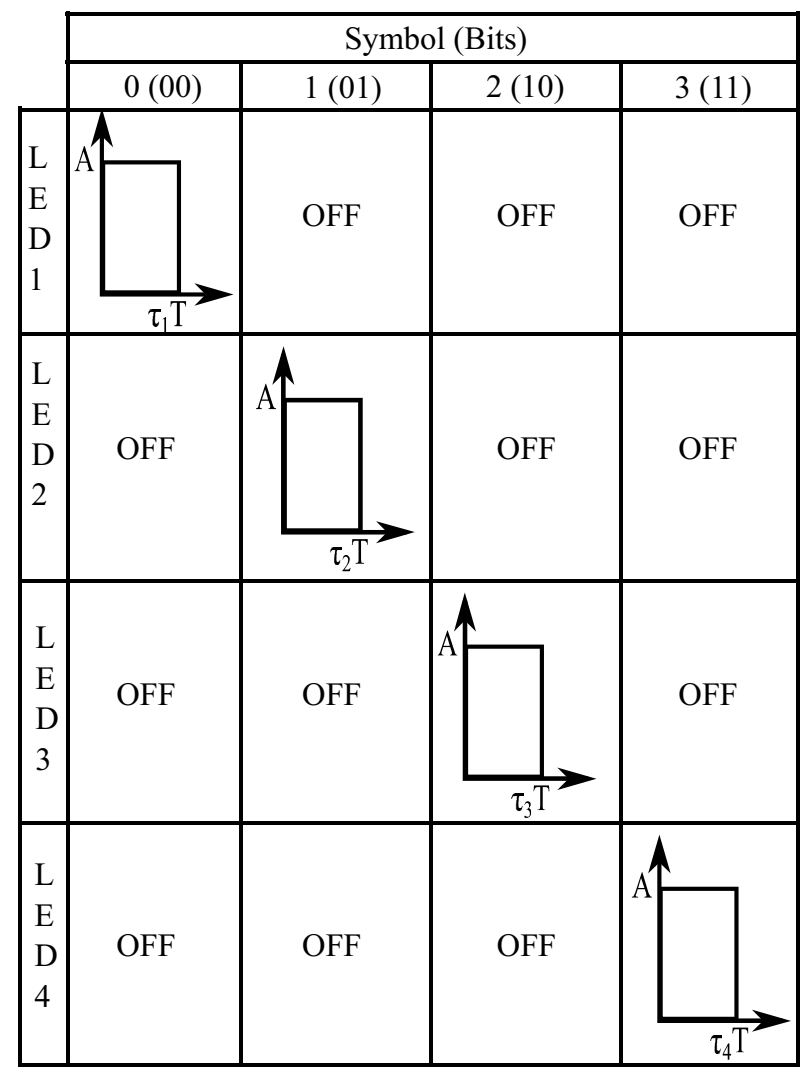

Fig. 1. The electrical pulse pattern for the enhanced SSK using variable pulse width.

responsivity of the photodetector (PD) is given as $R$ and the channel gain $\mathbf{H}(\mathbf{t})$ is:

$$
\mathbf{H}(\mathbf{t})=\left[\begin{array}{cccc}
h_{11}(t) & h_{12}(t) & \cdots & h_{1 N_{\mathrm{r}}}(t) \\
h_{21}(t) & h_{22}(t) & \cdots & h_{2 N_{\mathrm{r}}}(t) \\
\vdots & \vdots & \ddots & \vdots \\
h_{N_{\mathrm{t}} 1}(t) & h_{N_{\mathrm{t}} 2}(t) & \cdots & h_{N_{\mathrm{t}} N_{\mathrm{r}}}(t)
\end{array}\right]^{\mathcal{T}}
$$

where $h_{i j}(t)$ is the channel gain between the $i$-th transmitter and the $j$-th receiver and $\mathcal{T}$ represents the transpose operation.

For illustration purposes, if we consider a 4-LED system with $N_{\mathrm{r}}=1$, then the received signal $r(t)$ is:

$$
r(t)=s_{m}(t)+n(t)
$$

where

$$
s_{m}(t)= \begin{cases}h_{11} P_{\mathrm{t}} R ; & \text { for } m=0,0 \leq t \leq \tau_{1} T \\ h_{21} P_{\mathrm{t}} R ; & \text { for } m=1,0 \leq t \leq \tau_{2} T \\ h_{31} P_{\mathrm{t}} R ; & \text { for } m=2,0 \leq t \leq \tau_{3} T \\ h_{41} P_{\mathrm{t}} R ; & \text { for } m=3,0 \leq t \leq \tau_{4} T\end{cases}
$$

In (4), $P_{\mathrm{t}}$ represents the transmitted optical power from a source driven by an electrical signal of amplitude $A$ volts. By considering a correlator receiver architecture with a basis function $f(t)=\frac{1}{\sqrt{T}}$, thus the correlator output $\mathbf{r}_{\mathrm{b}}$ becomes: 


$$
\mathbf{r}_{\mathrm{bk}}= \begin{cases}h_{11} R P_{\mathrm{t}} \sqrt{T} \tau_{1}+n_{\mathrm{b} 1} ; & \text { for } m=0 \\ h_{21} R P_{\mathrm{t}} \sqrt{T} \tau_{2}+n_{\mathrm{b} 2} ; & \text { for } m=1 \\ h_{31} R P_{\mathrm{t}} \sqrt{T} \tau_{3}+n_{\mathrm{b} 3} ; & \text { for } m=2 \\ h_{41} R P_{\mathrm{t}} \sqrt{T} \tau_{4}+n_{\mathrm{b} 4} ; & \text { for } m=3\end{cases}
$$

where $n_{\mathrm{b} i}, i=1,2, \ldots N_{\mathrm{t}}$ is the Gaussian noise at the output of the correlator with variance $\sigma^{2}$. Using the maximum likelihood criterion, the estimated symbol $\hat{d}$ is obtained as:

$$
\begin{aligned}
\hat{d} & =\arg \max _{m} p\left(\mathbf{r}_{\mathrm{b}} \mid \mathbf{s}_{m}\right) \\
& =\arg \min _{m} D\left(\mathbf{r}_{\mathrm{b}}, \mathbf{s}_{m}\right)
\end{aligned}
$$

where the joint probability density function of $\mathbf{r}_{\mathrm{b}}$ conditioned on $\mathbf{s}_{m}$ being transmitted is given by:

$$
p\left(\mathbf{r}_{\mathrm{b}} \mid \mathbf{s}_{m}\right)=\frac{1}{\left(2 \pi \sigma^{2}\right)^{N_{\mathrm{r}} / 2}} \exp \left[-\sum_{k=1}^{N_{\mathrm{r}}} \frac{\left(r_{b k}-s_{m k}\right)^{2}}{2 \sigma^{2}}\right]
$$

The Euclidean distance metric $D\left(\mathbf{r}_{\mathrm{b}}, \mathbf{s}_{m}\right)$ is computed as follows:

$$
D\left(\mathbf{r}_{\mathrm{b}}, \mathbf{s}_{m}\right)=\sum_{k=1}^{N_{\mathrm{r}}}\left(r_{\mathrm{b} k}-s_{m k}\right)^{2}
$$

where $s_{m k}=\int_{0}^{\infty} s_{m}(t) f(t) \mathrm{d} t$, for $\quad k=1,2, \ldots N_{\mathrm{r}}$. The SER of the eSSKv can be derived by combining the pair-wise-error probability (PEP) with the union bound approach [13], [14] as follows:

Let $\mathrm{PEP}_{j \rightarrow i}$ be the probability of deciding in favour of symbol $i$ when symbol $j$ was transmitted, then:

$$
\begin{aligned}
\operatorname{PEP}_{j \rightarrow i} & =p\left(D\left(\mathbf{r}_{\mathrm{b}}, \mathbf{s}_{j}\right)>D\left(\mathbf{r}_{\mathrm{b}}, \mathbf{s}_{i}\right)\right) \\
& =p\left(2 \sum_{k=1}^{N_{\mathrm{r}}} n_{k}\left(s_{i k}-s_{j k}\right)>\sum_{k=1}^{N_{\mathrm{r}}}\left(s_{i k}-s_{j k}\right)^{2}\right) \\
& =Q\left(\frac{R P_{\mathrm{t}} \sqrt{T}}{2 \sigma} \sqrt{\sum_{k=1}^{N_{\mathrm{r}}}\left(\tau_{i} h_{i k}-\tau_{j} h_{j k}\right)^{2}}\right)
\end{aligned}
$$

Hence the symbol error performance union bound becomes:

$$
P_{\mathrm{e}, \mathrm{sym}}^{\mathrm{eSSKv}} \leq \frac{2}{N_{\mathrm{t}}} \sum_{j=1}^{N_{\mathrm{t}}-1} \sum_{i=j+1}^{N_{\mathrm{t}}} Q\left(\sqrt{\frac{\left(R P_{\mathrm{t}}\right)^{2} T}{4 \sigma^{2}}\left\|\tau_{i} h_{i k}-\tau_{j} h_{j k}\right\|_{\mathrm{F}}^{2}}\right)
$$

where $\|\cdot\|_{\mathrm{F}}$ is the Frobenius norm. In selecting the appropriate value of $\tau$ at the set-up stage, the objective is to maximise the value of $\left\|\tau_{i} h_{i k}-\tau_{j} h_{j k}\right\|_{\mathrm{F}}^{2}$. For the eSSKv, the average electrical signal-to-noise ratio (SNR) per symbol $\gamma_{\mathrm{eSSKv}}$ is defined as:

$$
\gamma_{\mathrm{eSSKv}}=\frac{\left(R P_{\mathrm{t}}\right)^{2} \sum_{i=1}^{N_{\mathrm{t}}} \tau_{i}}{N_{\mathrm{t}} \sigma^{2}}
$$

The average SNR for the SSK can be obtained from (11) by setting $\tau_{i}=1$ for all $i$.

\section{B. Optical SSK with Pulse Inversion (eSSKi)}

Although with an appropriate choice of $\tau_{i}$ it is possible to improve the error performance of SKK with the eSSKv, there exists the possibility of reduced spectral efficiency particularly when $\tau_{i}$ is small. To avoid this, the eSSKi can be used. It offers the same spectral efficiency as the SSK but with much improved error performance. This is achieved by increasing the effective distance between the constellation points. In effect, the eSSKi trades overall power efficiency for spectral efficiency. As shown in Fig. 2 for the case of $N_{\mathrm{t}}=4$ transmitters, eSSKi uses non-return-to-zero signalling just like the conventional SSK. But the even-indexed transmitters are driven with electrical pulses of amplitude $-A$ volts while the odd indexed ones have electrical pulses with amplitude $A$ volts. The essence of this is to increase the distance between adjacent constellation points.

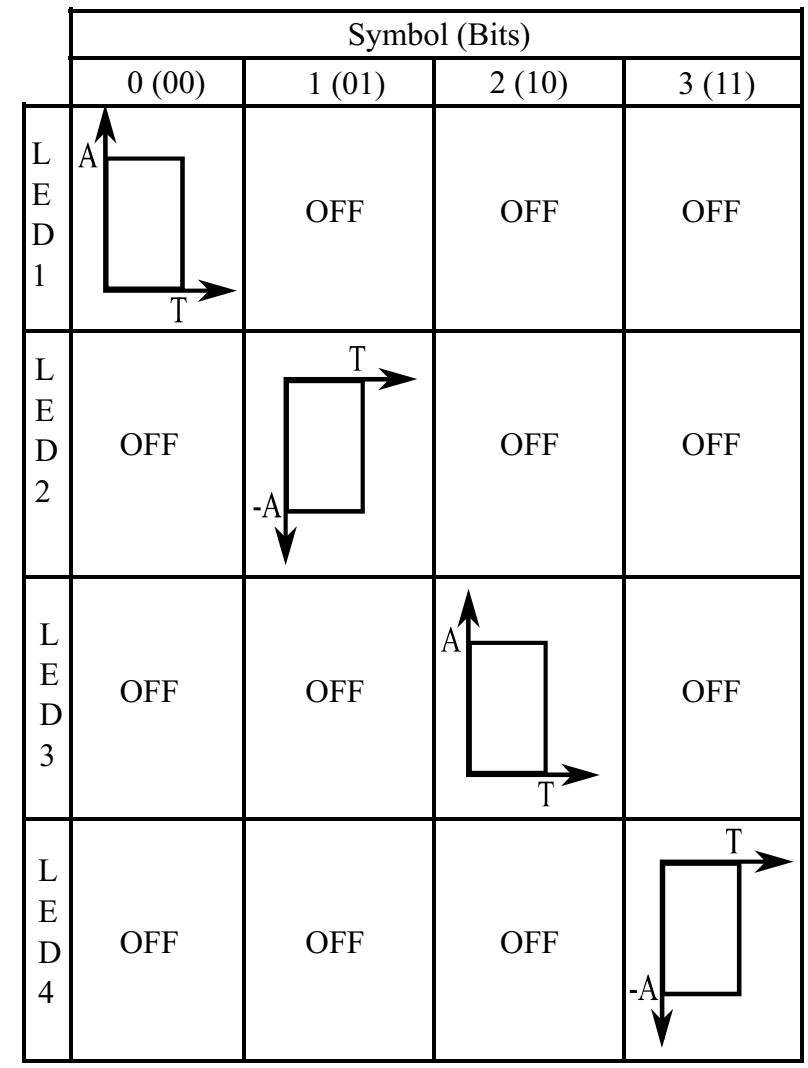

Fig. 2. The electrical pulse pattern for the enhanced SSK using pulse inversion.

Being a bipolar signalling technique, the eSSKi does require a DC bias. This requirement leads to a reduction in its overall power efficiency in comparison to the SSK. For VLC applications however, this is unlikely to be a disadvantage as the LEDs will have to be biased anyway to provide illumination.

By following the same approach as in the previous section, the SER of the eSSKi is derived as: 


$$
P_{\mathrm{e}, \mathrm{sym}}^{\mathrm{eSSKi}} \leq \frac{2}{N_{\mathrm{t}}} \sum_{j=1}^{N_{\mathrm{t}}-1} \sum_{i=j+1}^{N_{\mathrm{t}}} Q\left(\sqrt{\frac{\left(R P_{\mathrm{t}}\right)^{2} T}{4 \sigma^{2}}\left\|a_{i} h_{i k}-a_{j} h_{j k}\right\|_{\mathrm{F}}^{2}}\right)
$$

where $a_{l}=+1$ for $l=$ odd, otherwise $a_{l}=-1$. For the eSSKv, the average SNR per symbol $\gamma_{\mathrm{eSSKi}}$ is defined as:

$$
\gamma_{\mathrm{eSSKi}}=\frac{\left(R P_{\mathrm{t}}\right)^{2}}{\sigma^{2}}
$$

The two enhancement techniques of eSSKi and eSSKv make the conventional SSK adaptive. That is, the transmitter can choose the appropriate pulse feature based on the channel condition and the system constraints of power and spectral efficiency requirements.

\section{RESUlTS AND Discussions}

To evaluate the link performance, we will first be considering the benchmark conventional SSK. The results presented here will be based on an $N_{\mathrm{t}}=4$ and $N_{\mathrm{r}}=1$ optical wireless communication configuration. All the analytical results presented are also validated with Monte Carlo simulations. To demonstrate the link performance under identical channel gains, $\left\{h_{i 1}\right\}_{i=1}^{4}=[1,0.99,0.9,0.89]$ will be used while $\left\{h_{i 1}\right\}_{i=1}^{4}=[1,0.8,0.6,0.4]$ will be used for the case of uncorrelated channel.

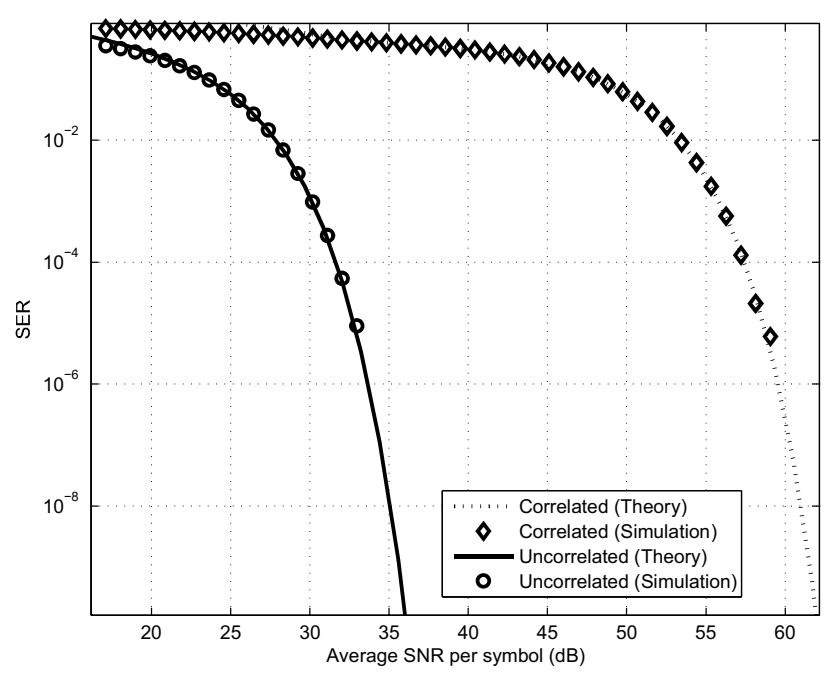

Fig. 3. The average SNR per symbol against the SER for conventional SSK with and without correlated channel gains.

From Fig. 3 where we show the SER of the conventional SSK at different SNR values, it can be observed that under the given parameters, the SER deteriorates with correlated channel gains. When the channel gains are correlated and very close, it becomes difficult to discern the transmitting LED at the receiver. For example at an SER of $10^{-6}$ and the specified channel gains, about $25 \mathrm{~dB}$ of extra SNR is required

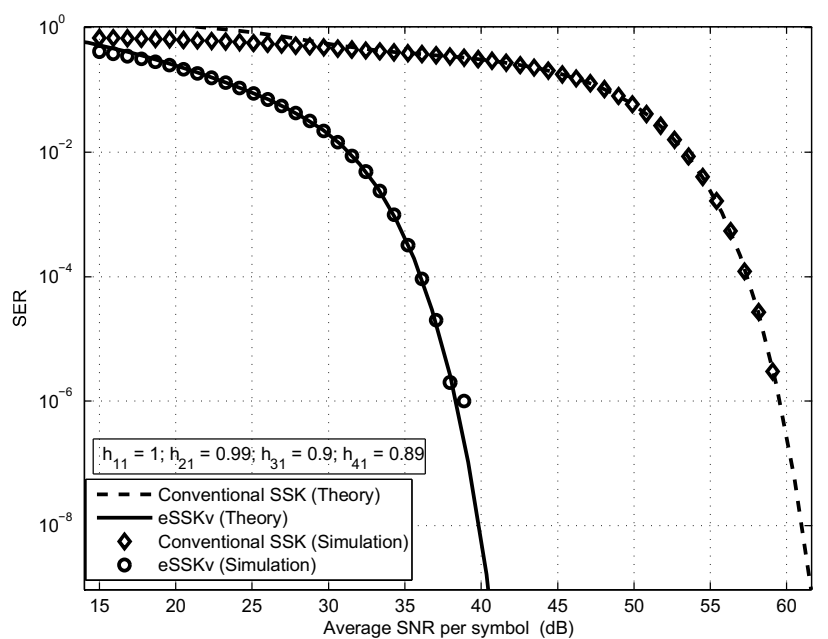

Fig. 4. The average SNR per symbol against the SER for four sources and one receiver: the enhanced SSK with variable pulse width with $\left\{\tau_{i}\right\}_{i=1}^{4}=$ $[1,0.8,0.6,0.5]$ and conventional SSK for $\left\{h_{i, 1}\right\}_{i=1}^{4}=[1,0.99,0.9,0.89]$

under correlated channel conditions to achieve the same error performance as when the channel gains are clearly distinctive.

To reduce this power penalty, we will now consider the enhancements of section III. For the variable pulse width enhancement, for any given set of $h i j$, the best of choice of $\tau_{i}$ is that which maximises $\left\|\tau_{i} h_{i k}-\tau_{j} h_{j k}\right\|_{\mathrm{F}}^{2}$. Based on this condition, we will be considering $\left\{\tau_{i}\right\}_{i=1}^{4}=[1,0.8,0.6,0.5]$ for the idential channel gains case. With these pulse width values, it is possible to reduce the SER required by SSK in correlated channel conditions by about $20 \mathrm{~dB}$ at an SER of $10^{-6}$ as Fig. 4 illustrates. This is brought about by the additional differentiation the variable pulse width introduces to the signals from the different LEDs. Under this channel correlation condition, the ultimate gain in terms of average SNR from the eSSKv enhancement depends strongly on the choice of $\tau_{i}$.

For the second enhancement technique, we show in Fig. 5 the error performance under the same closely-related channel gains. Here, considering the same SER of $10^{-6}$, a reduction in average SNR per symbol of about $18 \mathrm{~dB}$ could be achieved over the SSK. This is achieved by inverting the electrical pulse that feeds the even indexed tranmit LEDs. It should be highlighted that this type of enhancement requires an additional DC bias to make the inverted pulse positive. This inverted pulse technique can indeed be applied even when the channel gains are not so close as shown in Fig. 6. For the given channel gains in this figure, a gain of $7 \mathrm{~dB}$ in electrical SNR over the regular SSK can still be achieved at the benchmark SER of $10^{-6}$.

\section{CONCLUSION}

In this paper, an adaptive SSK modulation has been introduced to provide improved error over the conventional SSK in 


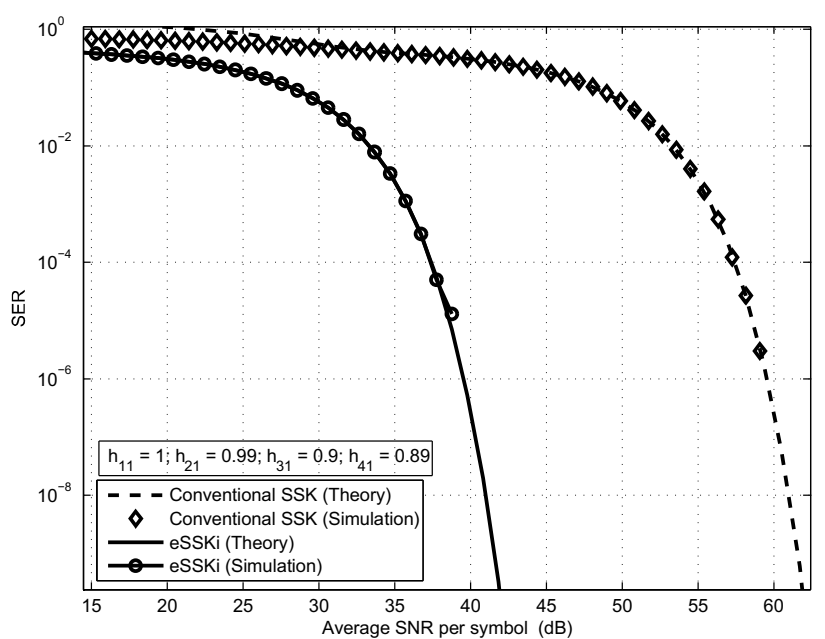

Fig. 5. The average SNR per symbol against the SER for four sources and one receiver: the enhanced SSK with pulse inversion and conventional SSK for $\left\{h_{i, 1}\right\}_{i=1}^{4}=[1,0.99,0.9,0.89]$.

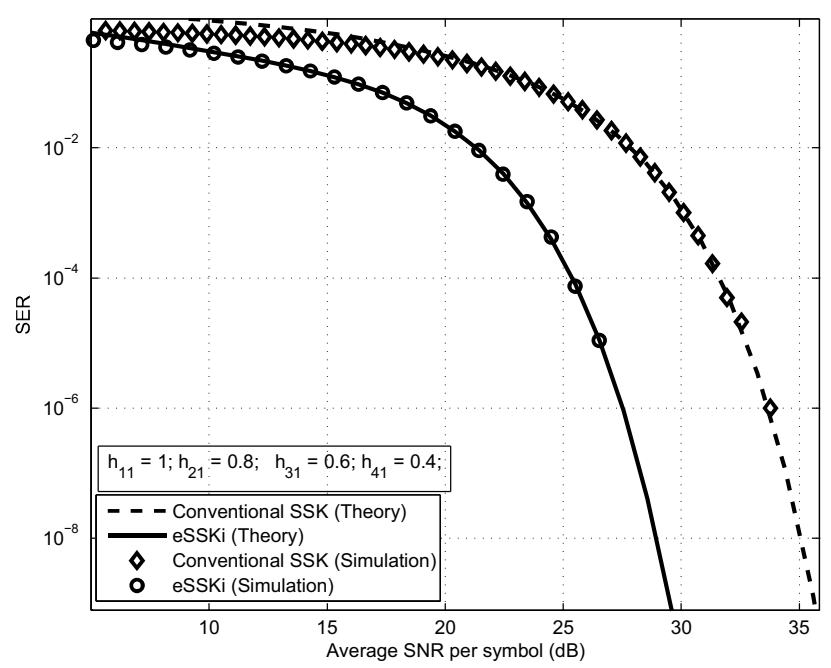

Fig. 6. The average SNR per symbol against the SER for four sources and one receiver: the enhanced SSK with pulse inversion and conventional SSK for $\left\{h_{i, 1}\right\}_{i=1}^{4}=[1,0.8,0.6,0.4]$.

the event of correlated channel gains. In one variant, different LEDs use different pulse widths (eSSKv) while the other approach uses pulse inversion (eSSKi) to offer resilience to channel correlation. Closed form expressions have also been given for the techniques and these have been verified with computer based simulations.

In a four-LED, one-receiver configuration with correlated channel gains $\left\{h_{i, 1}\right\}_{i=1}^{4}=[1,0.99,0.9,0.89]$, the enhancement techniques can produce a reduction of up to $20 \mathrm{~dB}$ in electrical SNR at and SER of $10^{-6}$ when compared with the conventional SSK.

\section{ACKNOWLEDGMENT}

This work is supported by research incentive grant (Trust Ref: 70004) from the Carnegie Trust.

\section{REFERENCES}

[1] W. Popoola and Z. Ghassemlooy, "BPSK Subcarrier Intensity Modulated Free-Space Optical Communications in Atmospheric Turbulence,' IEEE/OSA Journal of Lightwave Technology, vol. 27, no. 8, pp. $967-$ 973, April 2009.

[2] W. Popoola, Z. Ghassemlooy, J. Allen, E. Leitgeb, and S. Gao, "Freespace optical communication employing subcarrier modulation and spatial diversity in atmospheric turbulence channel," IET Optoelectronics, vol. 2, pp. 16-23, February 2008.

[3] L. Zheng and D. Tse, "Diversity and multiplexing: a fundamental tradeoff in multiple-antenna channels," IEEE Transactions on Information Theory, vol. 49, no. 5, pp. 1073-1096, May 2003.

[4] R. Mesleh, H. Haas, S. Sinanović, C. W. Ahn, and S. Yun, "Spatial Modulation," IEEE Trans. Veh. Technol., vol. 57, no. 4, pp. 2228 2241, July 2008.

[5] P. Yang, M. Di Renzo, Y. Xiao, S. Li, and L. Hanzo, "Design guidelines for spatial modulation," IEEE Communications Surveys Tutorials, vol. 17, no. 1, pp. 6-26, Firstquarter 2015.

[6] R. Mesleh, H. Haas, C. W. Ahn, and S. Yun, "Spatial Modulation A New Low Complexity Spectral Efficiency Enhancing Technique," in IEEE International Conference on Communication and Networking in China (CHINACOM), Beijing, China, Oct. 25-27, 2006, pp. 1-5.

[7] M. Di Renzo, H. Haas, A. Ghrayeb, S. Sugiura, and L. Hanzo, "Spatial Modulation for Generalized MIMO: Challenges, Opportunities, and Implementation," Proceedings of the IEEE, vol. 102, no. 1, pp. 56-103, Jan 2014.

[8] W. Popoola, E. Poves, and H. Haas, "Error Performance of Generalised Space Shift Keying for Indoor Visible Light Communications," IEEE Transactions on Communincations, vol. 61, no. 5, pp. 1968-1976, May 2013.

[9] J. Jeganathan, A. Ghrayeb, L. Szczecinski, and A. Ceron, "Space Shift Keying Modulation for MIMO Channels," IEEE Transaction on Wireless Communications, vol. 8, no. 7, pp. 3692-3703, Jul. 2009.

[10] R. Mesleh, H. Elgala, and H. Haas, "Optical Spatial Modulation," IEEE/OSA Journal of Optical Communications and Networking, vol. 3 , no. 3, pp. 234-244, Mar. 2011, ISSN: 1943-0620.

[11] W. Popoola, E. Poves, and H. Haas, "Spatial Pulse Position Modulation for Optical Communications," IEEE/OSA Journal of Lightwave Technology, vol. 30, no. 18, pp. 2948-2954, September 2012.

[12] W. O. Popoola and H. Haas, "Demonstration of the Merit and Limitation of Generalised Space Shift Keying for Indoor Visible Light Communications," Journal of Lightwave Technology, vol. 32, no. 10, pp. 1960-1965, 2014.

[13] J. G. Proakis, Digital Communications, 4th ed., ser. McGraw-Hill Series in Electrical and Computer Engineering, S. W. Director, Ed. McGrawHill Higher Education, December 2000.

[14] M. Di Renzo and H. Haas, "A General Framework for Performance Analysis of Space Shift Keying (SSK) Modulation for MISO Correlated Nakagami-m Fading Channels," IEEE Transactions on Communications, vol. 58, no. 9, pp. $2590-2603$, Sep. 2010. 